\title{
Pour une écopoésie de l'apprentissage de la lecture au cycle 2 , qui n'oublie pas les élèves en difficulté
}

"Écopoésie" to improve learning to read and help non-enhanced 6-8 years old students too

\section{Michel Favriaud et Dan Liu}

\section{OpenEdition}

\section{Journals}

Édition électronique

URL : http://journals.openedition.org/pratiques/5334

DOI : 10.4000/pratiques.5334

ISSN : 2425-2042

Éditeur

Centre de recherche sur les médiations (CREM)

Référence électronique

Michel Favriaud et Dan Liu, «Pour une écopoésie de l'apprentissage de la lecture au cycle 2, qui

n'oublie pas les élèves en difficulté », Pratiques [En ligne], 179-180 | 2018, mis en ligne le 31 décembre 2018, consulté le 01 mai 2019. URL : http://journals.openedition.org/pratiques/5334 ; DOI : 10.4000/ pratiques. 5334

Ce document a été généré automatiquement le 1 mai 2019.

(c) Tous droits réservés 


\title{
Pour une écopoésie de l'apprentissage de la lecture au cycle 2 , qui n'oublie pas les élèves en difficulté
}

\author{
"Écopoésie" to improve learning to read and help non-enhanced 6-8 years old \\ students too
}

Michel Favriaud et Dan Liu

1 D'après les résultats des deux enquêtes internationales, PIRLS, 2016 (Programme international de recherche en lecture scolaire) et PISA, 2012 (Programme international pour le suivi des acquis des élèves) - et en attendant ceux de l'enquête PISA 2019 - on constate qu'environ $20 \%$ des élèves français arrivent au collège sans maitriser la lecture et la compréhension des écrits et que la plupart des élèves français manifestent une appétence moyenne à apprendre, et ce malgré l'abandon, officialisé par étapes de 2002 à 2015, de la méthode semi-globale, censée responsable de l'échec - ce qui situe la France dans le milieu du tableau des cinquante pays étudiés. Or, s'il semble admis par presque tous que le non-déchiffrage automatisé obère la compréhension (Sprenger-Charolles, 1986, p. 9 et sq.), l'automatisation du code n'en est pas pour autant une condition suffisante. L'objectif des cycles 2 et 3 de l'école primaire et secondaire est donc d'assurer la fluidité de la maitrise du code et la compréhension-interprétation de textes (narratifs pour R. Goigoux \& S. Cèbe, 2006 ; 2013), tout en se posant la question de leur rapport.

2 Avec une bonne quinzaine d'années de recul, la question lancinante - puisque l'échec s'accroit malgré l'abandon de la méthode semi-globale (plus souvent seulement à départ global en GSM et au début du CP) - doit être revisitée et réactualisée : une application stricte (peut-être réductionniste ?) des données neuro-cognitives impose-t-elle un retour à des méthodes alphabétiques et combinatoires naguère jugées inefficaces et socialement discriminatoires ? S. Dehaene lui-même, référence nationale et internationale au plan de la psychologie cognitive, maintenant directeur du Conseil scientifique de l'Éducation 
nationale instauré par le ministre J.-M. Blanquer, se gardait bien, jusqu'il y a peu, de promouvoir une méthode précise d'apprentissage. Un simple ripolinage, comme il s'esquisse, par le jeu, l'informatique ou la robotique, ou même une transformation un peu plus profonde, par la remédiation différenciée ou par la préséance effective de l'écriture sur la lecture (Instructions officielles [IO] de maternelle 2015), envisagés maintenant, apureront-ils les comptes?

Car il y a non pas un, mais trois faisceaux de conditions impératives pour apprendre à lire de quatre à huit ans: 1) la maturation psychologique: désir de s'autonomiser et de grandir, estime de soi, amitié et curiosité envers la langue (Lebovici, Diatkine \& Soulé, 2004) ; 2) la construction des premiers schèmes piagétiens, qu'on a paru ne plus raccorder à la lecture depuis vingt ans; 3) la compétence linguistique, non seulement (méta)phonologique, mais aussi morphologique et sémantique (Gombert, 2003, p. 22-23). Notre hypothèse large est que la poésie, aux cycles 1 et 2 , pourrait faire le pont entre deux (sinon trois) de ces conditions majeures, et aussi entre apprentissages implicites et explicites (ibid., p. 28-29), alors que les IO (les IO de 2015), découvrant tardivement les sciences cognitives (mais peut-être pas les plus récentes relatives à l'émotion et à l'art), semblent, de nouveau, ne s'enivrer que d'une, de surcroit réduite à la phonologie (Blanquer, commission Dehaene, 2017).

4 Nous posons comme clé de voûte de notre proposition la contrainte sociale et politique, qui était au cœur des premières livraisons de Pratiques (Favriaud, 2016, p. 529-530) : les élèves en difficulté doivent pouvoir entrer dans la littératie, y vivre et progresser avec aise, tout en permettant aux autres d'avancer mieux (Liu, 2018 et ici même dans la partie III). En quoi est-ce que la poésie contemporaine pourrait satisfaire aux conditions psychologiques (1) et métalinguistiques (3) à la fois par définition et par ajustement didactique, sachant que les maitres du primaire ne sont pas des spécialistes de poésie?

On nous rétorquera peut-être que la poésie fait partie du socle républicain, sous les deux régimes successifs de comptines à la maternelle, de poèmes ensuite. Le passage des unes aux autres est-il pensé, construit? La récitation, genre d'activité (GA) canonique, est parfois (mais bien rarement) augmentée autour du $\mathrm{CP}$, voire dès la grande section maternelle (GSM), par un travail sur la voix et sur l'écriture "à la manière de »; ce premier GA ou le paquet des trois, n'ont pourtant, à notre connaissance, jamais été évalués dans leur rapport implicite, puis explicite avec l'apprentissage de la lecture.

Ou bien le problème vient-il d'une autre variable didactique, tue: le milieu scolaire d'apprentissage, lequel n'a qu'exceptionnellement été construit autour du nouage de la poésie et de la lecture ? C'est la question de l'« écopoésie » (Favriaud, 2016; Liu, 201811), comme milieu écologique scolaire d'apprentissage de la langue avec les arts et plus particulièrement la poésie. Il se pourrait que ce nouveau concept déborde largement l'ergonomie, pour toucher à l'éthique et au style, au sens redéfini par M. Macé (2016, p. 48-49), aux gestes professionnels de l'enseignant (Jorro, 1998 ; Favriaud, Vinsonneau \& Poletto, 2017, p. 230), en faisant sa juste place à l'imaginaire et la réflexivité linguistique et cognitive de l'élève.

7 Après les deux parties plus théoriques consacrées à l'articulation de nos concepts-clés, nous mènerons une enquête de terrain (Liu, 2018), de type ethnométhodologique (Coulon, 2014), en analysant des entretiens d'élèves, en difficulté et en réussite de lecture en GSM, au CP et au CE1. Notre défi, avec la poésie, est, sous certaines conditions matérielles, 
éthiques et professionnelles, de transformer les élèves les plus en difficulté et les autres, en lecteurs-poètes, heureux, habiles et réflexifs dans la langue.

\section{Poésie et apprentissage de la lecture : alliance naturelle ou stratégique?}

\section{La comptine, une petite-cousine bien utile en maternelle et au CP ?}

8 À entendre les enfants de maternelle réclamer une énième fois leurs comptines favorites, on peut penser que ces activités, engageant rythmiquement le corps-émotion de chacun, à sa guise, sont réconfortantes et stimulantes. Elles sont déclinées collectivement dans des activités d'oralisation, auxquelles on assigne couramment les finalités de retour au calme, de mémorisation, d'apprentissages notionnels divers (corporels, numériques, etc.) et d'approche phonologique implicite: repérage des sons, des rimes, de la place des syllabes, segmentation des mots, que les enseignants promeuvent depuis longtemps, bien que les IO 2015 de la maternelle n'en soufflent plus mot $^{2}$.

Mêlant plan imaginaire et plan linguistique, les comptines mettent en œuvre des refrains, des structures syntaxiques répétitives, et leur sens, souvent farfelu jusqu'au non-sens, fait humour (parfois noir), révélant une autre scène à demi cachée : «comptines d'enfance encore ondulatoires et circulaires et maniaques, revenances adorées de refrains, hantises inopinées de silhouettes, des aïeules, des monstres, des animaux, des arbres, des jardins, des morts » (Quignard, 2017, p. 143). Reliées à l'imaginaire archaïque et prélinguistique, elles permettent concomitamment d'entrevoir, accentuées par la voix et le corps, les composantes et les « problèmes » de la langue, au sens même d'É. Benveniste.

Les trois faisceaux de conditions ouvrant l'apprentissage de la lecture se mettent ici en place implicitement : 1) rythme de lallation, de bercement et de tension, qui rattache le sujet à son imaginaire profond et à celui des autres ; 2) travail sur le schéma corporel et la sériation ; 3) jeu épilinguistique tant au niveau du son, du lexique, de la syntaxe que de la sémantique. La réflexivité serait proche, si un adulte savait en mesurer le geste.

\section{La poésie, le genre littéraire le plus adéquat au soutien de la lecture?}

11 La poésie, par définition, évoque la vie dans ses origines, dans son tragique et ses émotions, dans ses intensités ; on pourrait dire que s'y joue le rapport entre la mort (ou du moins le manque) et la renaissance, qui est, on le verra tantôt, au cœur de l'imaginaire de tous, et peut-être l'un des enjeux de l'apprentissage de la lecture.

Ce rapport fantasmatique à la vie et au langage est figuré originalement en poésie par des lieux et des personnages émergents, quasi détourés, "proto-lieux» et "protopersonnages", qui sont les enveloppes fugitives que chacun peut investir subrepticement, dans des histoires à peine esquissées, souvent mêlées, que l'on appellera "proto-histoires» (réfutant ainsi la fausse antonymie entre poésie et récit), selon des temporalités elles-mêmes intermittentes ou flottantes. Le monde de la poésie n'est pas un monde mis au carré par la psychologie, la chronologie et les repères orthogonaux de la logique et du simili-réel, mais une masse souvent plus indécise, rythmée par des énergies et des intensités alternatives, dont les images (métaphores et comparaisons 
métaphoriques) sont les parangons insolites: mettant en tension deux éléments hétérogènes, suscitant une étincelle d'histoire, un lambeau de rêve, qui s'ancreraient dans un réel subjectif.

Si l'on compare maintenant la poésie avec d'autres genres ou sous-genres littéraires couramment utilisés depuis les années 1980, comme le conte, réputé « réparateur » pour les enfants en difficulté (Bettelheim, 1976; Diatkine, 1990), on voit que ce dernier est orienté vers des apprentissages apparemment plus structurants au plan psychologique: schéma corporel, personnage, lieu, chronologie, intrigue, résolution de problème, apprentissage de la vie, de la mort et du devenir adulte. Mais sans rappel de ce désordre imaginaire primitif, qui plait tant à certains enfants (Favriaud, Vinsonneau \& Poletto, 2017, p. 193), mais sans recours à la langue du bercement et de la destructurationrestructuration, qu'offre pourtant parfois une formulette: "Tire la chevillette et la bobinette cherra ", laquelle enchante les enfants en présentant concomitamment l'effroi de la langue archaïque énigmatique, et le réconfort de la comptine.

En revanche, la langue des poèmes et son rythme chaque fois singulier touchent aux couches les plus profondes de l'enfance par ses répétitions, refrains, rimes, allitérations et assonances, jouent et déjouent la signifiance, quasi sans référent explicite (Ricœur, 1975), par des incongruités, des jeux de mots, des polysémies, des suspensions - qui font lanterner - et des clausules - qui font couper court ${ }^{3}$. Nous comprenons alors que cette langue est une création négociée et négociable entre la "lalangue » lacanienne - faite de mouvements corporels, de manducations-succions, de lallations et de cris orientés vers le parent proche - la langue standard, symbolique, sociale, qui à l'écrit devient doublement symbolique, et donc plus abstraite encore, et une langue à venir plus pleine, telle que définie par E. Coseriu (voir les articles de C. Gérard et R. Missire ici même). Certains poèmes au moins, sinon tous, en rejouant probablement le rapport premier (dialogique sinon chaotique) au langage, réassureraient et dynamiseraient ainsi, par régression et progression, aux plans imaginaire, psychologique et cognitivo-schématique, les enfants les plus en difficulté comme tout un chacun.

Mais la poésie, éveille aussi, complémentairement, au fonctionnement lucide de la langue. Elle permet - par ses constructions, sa ponctuation élargie au blanc, au graphisme, voire aux accents (M. Favriaud, dans cette livraison), son anaphorisation généralisée (anadiplose, paragramme, paronomase), relevée tant par F. de Saussure que R. Jakobson, sa capacité aussi à s'inscrire durablement dans la mémoire - de retrancher pour les considérer de près les constituants minimaux du discours : phonème, syllabe, mot isolé de la linéarité phrastique, syntagme faisant rejet ou contre-rejet, vers et phrases, structurations syntaxiques. Elle permet aussi de développer le questionnement sémantique, toutes les fois que le poème sort des lignes syntaxiques standards et use de détours - symboles, paraboles, fables, métaphores - pour faire et défaire du sens. Ainsi la poésie provoque-t-elle implicitement dans le langage un «effet loupe » et un "effet question", qui préparent à une réflexivité sur la langue et son fonctionnement indispensable à l'apprentissage de la lecture, selon J.-É. Gombert (2003) et les cognitivistes en général.

Le passage de l'imaginaire à la réflexivité linguistique et discursive ne se fait pas directement, mais par étayage adulte (Vygotsky, 1997). Les comptines et la poésie, plus et autrement que contes et albums, pourraient être des aides didactiques précieuses, mais à des conditions, définitionnelles et pragmatiques, qu'il faut poser maintenant. 


\section{Prendre l'« imaginaire » comme moteur de la lecture}

\section{Un concept central mal défini}

17 Le mot « imaginaire » se révèle comme un des plus insaisissables en sciences humaines, philosophie, littérature et didactique, utilisé deux fois dans les IO de l'école maternelle 2015 (mais semble-t-il avec deux sens différents). L'adjectif, lui, se réfère tant à l'imaginaire qu'à l'imagination, dans ses deux faces, reproductrice ou créatrice (Duborgel, 1984). C'est assez dire qu'imagination et imaginaire sont souvent confondus, laissant le second sans caractérisation ni valeur spécifique, tant pratique qu'heuristique.

Plutôt que de repasser par les travaux fondateurs de G. Bachelard, G. Durand ou J. Burgos sur l'imaginaire, nous voudrions d'abord prendre en compte les travaux d'anthropologie de l'écriture d'A.-M. Christin (1995; 2011) sur la comparaison des systèmes alphabétiques et idéographiques, et la perte occasionnée, selon elle, par le choix alphabétique, certes plus économique, mais coupant l'humain de sa part sensible, esthétique, voire métaphysique. Ce n'est pas un hasard si cette anthropologue a cherché dans les poèmes de S. Mallarmé et de P. Reverdy, et leur utilisation du blanc dans la page, des façons de remotiver les signes et de réiconiser les textes (M. Favriaud dans cette livraison), nous ouvrant une voie épistémologique et didactique.

\section{Reconfiguration du concept par les écrivains-poètes contemporains}

19 On remarque dans la production littéraire des dernières années en France que nombre d'écrivains, poètes à différents degrés, font de «l'imaginaire» le noyau explicite d'œuvres tardives, en employant expressément le substantif comme Y. Bonnefoy (2016), F. Cheng (2016), A. Herbauts (2006), ou en l'évitant, comme P.Quignard (2017) ou P. Chamoiseau (2016). Tous semblent mettre en scène ce "passage " ou «transitoire » (nommé ainsi et exploré par H. Michaux), entre inconscient, création et réflexivité. Considérons ici plus particulièrement les trois derniers.

Pour P. Quignard, dans Performances de ténèbres (2017, p. 142) la scène traumatique configurante serait celle du passage de la vie fotale à la vie mondaine autonome, dont le théâtre, le nô japonais par excellence, mettrait en scène l'évènement par noir et blanc et dont l'architecture théâtrale serait la primitive grotte. Tout laisse à penser que l'entrée dans le langage social oral à dix-huit mois serait une seconde rupture, comme le suggère J. Lacan.

21 Pour P. Chamoiseau (2016, p. 16), l'homme est partagé entre un imaginaire singulier, bâti sur «les lumières orphelines après le cocon utérin ", le "creusement qui se nourrit de lui-même», les "pertes» "ouvrant d'étranges passages»; et un (ou plusieurs) imaginaire collectif, lié chez lui à la culture créole post-esclavagiste et sa croyance aux zombis (ibid., p. 20-23). L'énergie de la création naitra du rapport plus ou moins agonistique entre ces deux principales instances de l'imaginaire.

A. Herbauts, dès la première page de son album de jeunesse, De temps en temps (2006), part en quête explicite du rapport entre l'imaginaire d'une part, et la création poïétique et réflexive d'autre part :

de temps en temps,

je me retrouve à réfléchir sur le dire, sur le faire, sur le taire. 

effets pathiques :

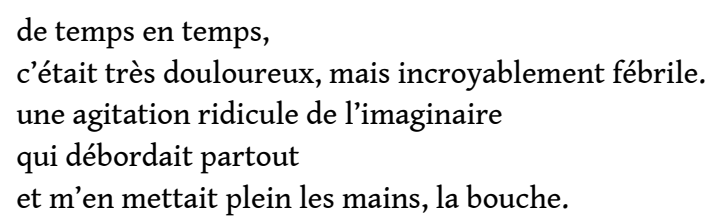

de temps en temps,

c'était très douloureux, mais incroyablement fébrile. une agitation ridicule de l'imaginaire qui débordait partout et m'en mettait plein les mains, la bouche.

A. Herbauts met ainsi au jour, tant par les mots et les phrases souvent segmentées par la ponctuation blanche, que par les illustrations en vis-à-vis, une symptomatologie : fièvre, agitation, rire, débordement, malpropreté, désordre, que les enseignants de maternelle et de primaire reconnaitront; accompagnée d'une pathologie: fièvre, douleur; et d'une étiologie :

de temps en temps, mes attaches me faisaient mal, mais je ne parvenais pas à les couper.

les amarres de la vie sont solides : je les entendais grincer contre la cale. leur écho difforme.

On pourra mettre celle-ci en relation avec cette deuxième double page, euphoriquedysphorique :

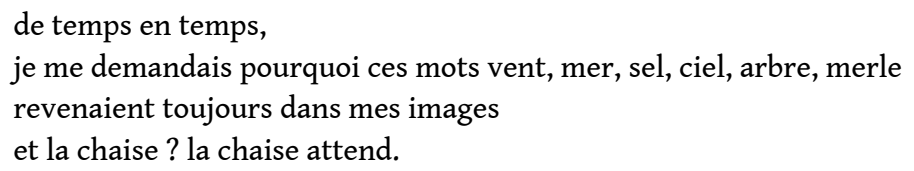

26 C'est le passé, la famille, l'enfance qui cognent douloureusement, et plus encore l'absence et le manque, métonymisés par la chaise vide. Les images naturelles, euphoriques, d'un corps heureux et rassemblé (celui de la mère enceinte ?), sont traversées et questionnées ( "me demandais pourquoi », « revenaient toujours dans mes images ») par leur contraire, chaotique et monstrueux, comme le suggèrent l'illustration enfantine, maladroite, inquiétante du rhinocéros à la tache rouge, et la phrase déchiquetée en enfilade verticale :

de temps/ en temps,/ on ne/ comprend/ plus/ quelle place/ prend/ le réel./ «le réel » évoqué comme subjectif, incompréhensible, insondable, source de quête et de désir, s'opposant ici à la « réalité » (voir page citée infra). L'écriture se situe ainsi entre l'angoisse de langage - écartelé entre l'idiosyncrasique et le social :

de temps en temps,

je criais pour savoir si je savais encore parler.

parler en dehors de moi,

en dehors de ce couloir qui longe le texte et l'image,

peut-être allais-je devenir folle? je ris.

28 - et la levée salvatrice des formes et idées, qui retourne la terreur en énergie momentanément positive :

de temps en temps, on essaye de se concentrer, de capturer ces pensées informelles qui changent sans cesse de consistance, on a parfois la vague impression de pouvoir écrire - en une image - la mer. mais le ressac du papier a vite fait de nous rejeter dans la réalité.

On aura reconnu en passant le rôle du rythme dans l'écriture d'A. Herbauts, relié à la fragmentation et à la fréquentativité du temps - non diégétisé ni vectorisé, oscillant entre imparfait et présent dilaté, mais suturé par le quasi-refrain de la formulette temporelle, 
agencée différemment dans chaque double page, de gauche, de droite, en début de laisse ou une fois à la fin. l'imaginaire :

a. A partie liée avec la naissance, la petite enfance, la mort, le manque ;

b. est relié consubstantiellement au langage linguistique et iconique, mais autant à son surgissement, son bégaiement (G. Luca) qu'à son résultat stabilisé (A.du Bouchet dans L'Incohérence);

c. peut avoir des sources singulières ou collectives;

d. crée de la douleur, de l'agitation, de l'inquiétude, pouvant entrainer un questionnement sur soi, sur l'enfance, mais aussi sur les formes sémiotiques;

e. crée de l'énergie bipolaire, négative et positive; opère un retournement continuel des polarités ; en cela source de vie, de transformation ;

f. écriture et invention créatrice sont ses modes d'expression, de symbolisation et de réflexivité privilégiés ;

g. anamorphose, métamorphose, métaphore et anaphore sont ses figures privilégiées, qu'il partage avec le rêve et la poésie ;

h. rythme et prosodie, comme construction d'un temps à la fois régulier, ressassant, et irrégulier, elliptique, chaotique, en sont les formes évocatrices, intersubjectives et heuristiques.

\section{Hypothèses affinées du rapport possible entre didactique de la poésie et didactique de la lecture-écriture au cycle 2}

31 L'apprentissage de la lecture-écriture correspondrait au passage du langage oral, du corps-émotion de la petite enfance, au langage écrit, normé, décomposé, analysé, recombiné, sous l'injonction supérieure, relayée par les parents, du « devenir grand ». Si on ajoute que certains élèves sont partagés entre deux langues, maternelle et scolaire, et deux cultures et systèmes de croyance, ce deuxième saut symbolique pourrait être durablement traumatique pour plus d'un.

La poésie contemporaine pourrait opérer chez les élèves de GSM, CP, voire CE1, un triple effet : un " effet réparation » sur l'image de soi, un " effet loupe » et " un effet question » sur leur rapport à la langue de l'écrit; elle aurait l'avantage, quasi définitionnel, de réaccorder harmonieusement le corps-émotion et le cerveau analytique et réflexif. Le passage par le réflexif, donc l'abstraction, quand il est coupé de son imaginaire d'enfant et de l'étayage perspicace du maitre, ne risque-t-il pas d'inhiber beaucoup d'enfants, sinon, à des degrés différents, tous?

Auraient aussi un grand rôle à jouer dans cette didactique enrichie de la lecture : le choix des activités didactiques de poésie, l'étayage différencié du maitre, et le milieu d'apprentissage (Favriaud, Vinsonneau \& Poletto, 2017 ; Liu, 2018), quasi seconde peau, au sens de D. Anzieu (1994).

La dernière série de questions vise le rapport entre activités de poésie et méthode de lecture stricto sensu: une méthode d'apprentissage systématique de type synthétique serait recommandée, mais à la condition expresse que les activités de poésie, durables et renouvelées, non seulement l'accompagnent mais soient explicitement mises en rapport avec ledit apprentissage de la lecture et de la langue. Ainsi le maitre aurait-il moins 
besoin d'être spécialiste de poésie que d'être investi dans une éthique plus générale de la création et de la réflexion.

\section{Prolégomènes à une écopoésie de la lecture}

\section{Des comptines aux poèmes : rupture ou continuité avec la lecture?}

J.-M. Gauthier et C. Lejeune (2008, p. 414) affirment que le « chant » apporte aux enfants une "sécurité communicative", améliorant leurs performances langagières et lexicales. Des chercheurs cognitivistes ont en outre démontré l'effet positif des comptines sur la sensibilité phonologique de ceux-là (Bryant et al., 1989, p. 407). L'usage et la manipulation des comptines s'avèrent un bon prédicteur d'apprentissage de la lecture et du code. Toutefois ces avantages sont peu et irrégulièrement promus par les IO, qui remisent les comptines, tout comme la poésie au cycle 2 , dans les pratiques de l'oral, alors que la poésie par définition ferait le pont entre oral et écrit, ce que suggère indirectement la définition meschonnicienne de l'oralité de la poésie écrite, du côté du rythme (Meschonnic, 1995) et des syntaxes plurielles (Favriaud, 2014). Or l'un des nœuds de l'apprentissage de la lecture, n'est-ce pas ce passage harmonieux et réfléchi de l'oral à l'écrit?

Ainsi, dans les IO comme dans les pratiques professionnelles ordinaires, le bénéfice constaté des comptines est triplement minoré, par l'oubli des comptines à l'école élémentaire, par le manque de tuilage entre comptines et poèmes, et par la dissociation radicale entre poésie et apprentissage de la lecture au cycle 2 (Liu, 2018, p. 23). Des activités pertinentes de poésie au primaire - par-delà la sacro-sainte récitation - ne pourraient-elles pas au contraire assurer le continu des apprentissages, de l'émotionnel au réflexif, permettre une différenciation plus fine des sujets apprenants, et donc des remédiations qui ne se limiteraient pas à la seule compétence métaphonologique isolée de tout contexte?

\section{Les élèves de CP et de CE1 se différencient-ils selon leur degré de littératie ou de façon plus singulière?}

Notre échantillon a été constitué dans des classes de $\mathrm{GSM}^{4}$, de CP et CE1 de la grande banlieue toulousaine, socialement diversifiées, conduites respectivement par un maitre expérimenté non expert de poésie, un maitre expert appartenant au groupe Apprentissage de la lecture et poésie (Alep) et au Groupe français d'éducation nouvelle $(\mathrm{GFEN})^{5}$, et un maitre inexpérimenté en poésie mais s'y intéressant fort.

Tableau 1. Profil de l'échantillon

\begin{tabular}{|c|c|c|}
\hline Classe & Élève & Compétence de lecture-déchiffrement \\
\hline \multirow[t]{2}{*}{ GSM } & $\mathrm{E}$ & Avancé \\
\hline & $\mathrm{T}$ & Suivi par un orthophoniste \\
\hline CP & B & Normal mais lent \\
\hline
\end{tabular}




\begin{tabular}{|l|l|l|}
\cline { 2 - 3 } & C & Avancé \\
\hline \multirow{4}{*}{ CE1 } & P & Suivi par un orthophoniste \\
\cline { 2 - 3 } & L & Normal \\
\cline { 2 - 3 } & $\mathrm{J}$ & Expert \\
\hline
\end{tabular}

Les élèves retenus ont eu un entretien semi-directif avec le chercheur. En GSM, la poésie a été très présente au premier trimestre sous l'aspect de la récitation et du cahier de vie, soit trois mois avant l'entretien, tandis qu'en CP-CE1, celui-ci a eu lieu dans la suite d'un entrainement continu à la poésie de plus d'un semestre, passant par des activités de diction et de production créatrice.

Tableau 2. Activités de poésie pratiquées

\begin{tabular}{|l|l|l|l|}
\hline \multicolumn{2}{|l|}{ POÉSIE (CP, CE1) } \\
\begin{tabular}{|l|l|l|l|} 
Activités \\
quotidiennes \\
ritualisées
\end{tabular} & $\begin{array}{l}\text { Lecture et copie } \\
\text { chaque matin d'un } \\
\text { extrait de poème }\end{array}$ & $\begin{array}{l}\text { Débat interprétatif } \\
\text { après mémorisation } \\
\text { du poème }\end{array}$ & $\begin{array}{l}\text { Recherche et lecture d'un } \\
\text { album de poésie dans la } \\
\text { classe ou à la bibliothèque } \\
\text { communale }\end{array}$ \\
\hline $\begin{array}{l}\text { Activités } \\
\text { semestrielles }\end{array}$ & $\begin{array}{l}\text { lorrespondancer de } \\
\text { ésie avec d'autres }\end{array}$ & Correspondance de poésie avec des poètes \\
\hline $\begin{array}{l}\text { Activités } \\
\text { annuelles }\end{array}$ & $\begin{array}{l}\text { Création } \\
\text { présentation d'un } \\
\text { spectacle de poésie }\end{array}$ & Ateliers de poésie avec des poètes \\
\hline
\end{tabular}

À partir de ces entretiens nous pouvons établir les rôles que les élèves attribuent à la poésie, comme suit.

\section{Le renversement thymique}

Pour C de CP, l'effet apaisant de la poésie est exprimé très explicitement :

- D : La poésie te plait?

- B : Oui.

- D : Comment? Tu te sens mieux quand tu lis la poésie?

- B : Oui. Parce qu'à chaque fois le soir... ça me fait un peu énerver et je n'arrive pas

à dormir. Et à chaque fois la poésie ça me calme.

41 L de CE1 explique bien l'effet thymique de la poésie dans toute la gamme des émotions, tant euphoriques que dysphoriques, et en fait même la finalité épistémologique de cet art du langage :

- D : Pour toi, la poésie sert à quoi?

- L : Ca sert à exprimer des choses.

- D : Et toi, tu exprimes quoi?

- L : Par exemple, les émotions. Quand je suis en colère, j'exprime mes colères. 
Quand je suis triste, j'exprime la tristesse.

- D : Quoi d'autre?

- L : La peur, la joie... librement, déclare au chercheur :

- D : Ce poème ça parle de quoi ?

- T : Ca parle d'un enfant, il a trouvé son passé et son avenir.

- D : Et après?

- $\mathrm{T}$ : Après il pourra revenir dans son passé ou dans son futur.

- D : T'aimes bien être cette personne ? C'est toi ?

- T : Pour moi, oui.

- D : Et t'aimes bien retourner au passé ?

- $\mathrm{T}$ : Oui.

- D : Et pas au futur?

- T : Non, je ne sais pas ce qui m'attend. Moi je préfère en bas.

$\mathrm{T}$ suggère que les apprentissages scolaires et la vie futurs lui causent de l'incertitude, sinon de la panique. Toutefois le chercheur remarque le calme et la prudence de $\mathrm{T}$ pendant tout l'entretien, où il exprime à la fois la souffrance causée par la poésie qui le met au défi de grandir, et le soulagement de pouvoir extérioriser et fixer sur un autre personnage son angoisse d'apprendre. Cet effet contra-thymique corrobore les observations de M. Vinsonneau dans Les chemins de poésie d'Alep (Favriaud, Vinsonneau, \& Poletto, 2017, p. 181), quand elle utilise, parallèlement à l'apprentissage de la lecture au $\mathrm{CP}$, le livre de $\mathrm{S}$. Creech, J'aime pas la poésie, mettant en scène un apprenant rebelle, qui fait peu à peu son chemin d'Alep. Dans l'apprentissage de la lecture, ne faut-il pas, d'une certaine façon, renverser les émotions et faire une conversion?

Nous remarquons en outre que le poème aide $\mathrm{T}$ à construire le schème piagétien de la temporalité et à mieux situer sa vie présente entre passé refuge et avenir plus incertain mais néanmoins envisagé, que le poème aidera peut-être à anticiper de façon plus sereine.

Nous voyons donc que la poésie peut à la fois figurer et "proto-narrativiser » les émotions des apprenants (ce qui engage les praticiens à choisir des poèmes s'y prêtant), et opérer un « effet de réparation » en assignant à celles-ci, par le langage et le rythme du poème, un nom, une place, un retournement et une esthétisation. L'effet de réparation des «émotions tristes " par la poésie ne se limiterait pas au plan psychologique, mais renforcerait les schèmes cognitifs des apprenants et ouvrirait, bien plus que les «purs » récits (non poétiques), à l'écoute du langage, à la confiance qu'on peut lui accorder, et à la curiosité qu'occasionne sa dimension polysémique.

\section{De l'émotion à l'imagination}

L'émotion en poétique et en réception de poésie s'abouche à l'imagination, tant reproductrice que créative. Narrativiser le poème, comme le font les enfants - ce qui peut laisser pantois certains maitres formés à la distinction radicale des genres littéraires faire ou vivre les métaphores, c'est entrer dans l'imagination reproductrice et l'imagination créatrice, de frontière poreuse. M. Favriaud et al. (2011) avaient déjà pressenti que les élèves qui, au cycle 2 , manifestaient l'imagination créatrice la plus surprenante, apparemment délirante, n'étaient pas, en fait, les moins avancés dans le rapport entre poésie et apprentissage de la lecture et de la langue ; ils investissaient plus 
de leur imaginaire singulier, faisaient un effort pour le narrativiser, et prenaient des risques, qu'il vaudrait mieux accompagner que censurer.

Dans le corpus de $\mathrm{D}$. Liu, on relève cependant un troisième type d'imagination qui fait lien avec la symbolisation écrite et la réflexivité sur la langue écrite - que l'on pourrait appeler l'imagination scripturale et linguistique. Ainsi P de CE1 déclare-t-elle au requérant:

- D : Est-ce que tu penses que l'ordre des mots est important dans la poésie?

- $\mathrm{P}$ : Non.

- D : Pourquoi?

- P : Parce qu'on pourrait mettre les mots partout. Par exemple, en fait s'il y a des mots qui sont à travers, je les remets droit dans ma tête.

- D : Ah, oui, automatiquement?

- P : Beh, je pense. Alors lettre droit, j'imagine le texte. Et ça me fait les remettre droit ${ }^{6}$.

- D : Et l'ordre des mots est important dans le récit, histoire par exemple?

- P : Oui.

À cette étape-là, l'activité mentale de l'élève n'est plus centrée sur l'imagination lyrique ou narrative du poème, mais bien sur le code linguistique par comparaison de deux régimes de langue, le régime lyrique, plus libre et créateur, et le régime narratif standard. On pourrait dire ici que cette élève, pourtant identifiée par l'institution comme élève en difficulté, fait déjà le rapport entre poésie et langue au niveau syntaxique, en établissant un tableau mental comparatif, ce qui est une compétence de haut niveau même au cycle 3. Les activités créatrices de poésie, qu'on croyait uniquement liées à l'émotion et à l'imagination fantasque, suscitent, dans ce genre particulier d'activité qu'est l'entretien didactique, dûment étayé, des réactions cognitives insoupçonnées, qui renversent les évaluations traditionnelles et la notion d'échec scolaire.

\section{Les compétences de déchiffrage et de réflexivité activées}

$C$, élève de $\mathrm{CP}$, est entrée dans la lecture de façon satisfaisante mais, scrupuleuse, demande toujours du temps supplémentaire pour accomplir les tâches, ce qui parfois l'exaspère et entame sa confiance. Voici l'échange entre les deux partenaires d'entretien :

- D : Est-ce que tu penses que la poésie, ça va t'aider à lire?

- C : Oui, en fait des fois, le maitre nous donne un poème pour lire à la maison qu'on a déjà révisé plein de fois et en fait maman... je lui dis : j'ai un poème à lire ; je prends mon cahier sans mon cahier [sic], je lis ça dans deux minutes. Ca veut dire que ça aide à lire.

Mimant la scène traditionnelle de lecture à la mère, elle déclare qu'à force de répéter le poème, elle peut le réciter par cœur, en simulant la lecture dans le cahier. Bien qu'elle confonde peut-être récitation et lecture (ce qu'on a souvent reproché à la méthode à départ global), on peut être sûr que la mémorisation occupe un rôle essentiel dans son apprentissage de la littératie. C'est du moins la solution consciencieuse qu'elle a trouvée pour gagner de la confiance. $\mathrm{C}$ fait donc bien le rapport entre poésie et apprentissage de la lecture; mais le maitre devra corriger cette stratégie d'apprentissage de la lecture globalement erronée.

$51 \mathrm{~T}$, l'élève en difficulté au $\mathrm{CP}$, s'efforce de déchiffrer les mots du poème qu'il préfère: notamment le mot « ruisselle », qui semble stimuler son imaginaire :

- D : D'autres?

- $\mathrm{T}$ : Après il y a des majuscules. Après tu vois deux ss ça ne fait pas de /z/ (dans

" ruisselle »), ça fait de /s/. Après c avec e, ça fait /ce/. 
- D : Oui d'autre?

- T : U, i qui fait /e/, et elle.

52 À notre stupéfaction, $\mathrm{T}$ établit les règles de la combinatoire : le « ss », différent du simple « $\mathrm{s}$ » pour faire le son [s], le « $\mathrm{c}$ » associé au « e » pour faire le même phonème. Même si la combinaison $d u$ « $u$ » et $d u$ « $i$ » aboutit à une erreur, sans doute par confusion de la première et dernière syllabe sonore du même mot, on retiendra que la poésie ne détourne pas l'élève des lois de la combinatoire, mais au contraire l'excite à les chercher. Contrairement à $\mathrm{C}$, $\mathrm{T}$ semble utiliser la bonne stratégie.

53 À la question du chercheur: "Est-ce que les erreurs de diction de tes camarades te gênent?", L répond que "ça pouvait être beau de faire des erreurs ", ajoutant que les poèmes pouvaient en tirer de la beauté supplémentaire; elle paraphrasait ainsi le jugement esthétique et quasi épistémique du maitre disant qu'en poésie, genre où les éléments étaient plus libres, l'erreur parfois suscitait une belle création personnelle. Ce dialogue permet de faire une quadruple hypothèse : 1) l'assimilation par les élèves d'un ethos de l'apprentissage promu par le maitre serait accrue par une telle activité ; 2) leur sentiment de crainte face aux apprentissages serait allégé, voire retourné, non seulement par l'activité centrale de poésie, mais par la présence empathique des pairs, (Liu, 2018) ;3) cette élève de bon niveau de $\mathrm{CP}$ est parvenue à un taux de tolérance et d'abstraction lui évitant des jugements émotionnels négatifs ; 4) elle est capable sinon d'une définition de la poésie, du moins d'une affirmation de certaines de ses caractéristiques. Ce niveau émotionnel et réflexif pourrait être un bon prédicteur de littératie.

54 E est une élève de GSM où la récitation prend la suite des comptines, sans que d'autres activités d'écriture ou de diction soient mises en œuvre, sinon un cahier de vie, qui circule de la classe à la maison, créant un élément d'écopoésie. Cette élève va se livrer à une prouesse de lecture qui témoigne à la fois de son entrée en littératie et de sa capacité à jouer avec les stratégies de lecture, assignant ainsi aux poèmes de multiples possibilités de réassemblage et parcours lectoraux. Cela témoigne d'une pratique de la syntaxe plurielle (Favriaud, Vinsonneau \& Poletto, 2017) et de la signifiance $n^{\circ} 2$, telle que définie par R. Missire dans cette livraison même, les deux étant caractéristiques du genre poétique :

- D : Qu'est-ce que tu remarques dans ce poème?

- E : Pourquoi il y a trois «ils », l'un après l'autre ? En dessus de l'autre. C'est fait exprès... si on lit comme ça, « ma je dans un grégoire peut-être on ils hou » (elle a lu tous les premiers mots des vers)

- D : Et après, comment tu lis ?

- E : Après on peut lire comme ça : «amoureux deux bisous cou partout pou serré

l'été amoureux Mathieu deux cou doux et hou » même si... et hou c'est le premierdernier!

- D : Il y a d'autres choses que tu remarques dans ce poème?

- E : Non... c'est aussi on peut lire comme ça !!(rire)

- D : C'est rigolo! À l'envers!

- E : Et aussi comme ça!

- D : Tu veux essayer à lire?

- E : «Amoureux un a sœur ma»

- D : C'est rigolo!

55 Cette élève tellement avancée en GSM non seulement a lâché prise grâce au poème, mais s'est aventurée dans la lecture tous azimuts du poème, sans négliger ni le déchiffrage, ni le repérage morpho-sémantique du « ils » anaphorisé. Elle n'en tire certes pas de grandes conséquences sémantiques, mais déjà un jugement de goût, proposé par le chercheur ( 
«C'est rigolo ! ») qui est l'amorce d'un jugement de valeur esthétique et d'une approche épistémologique de la poésie. On perçoit de nouveau l'intérêt didactique majeur de l'entretien d'élucidation didactique duel, mené par un adulte qui lui-même a lâché prise et suit, avec enjouement et lucidité (voir kaïros et bonification ci-après) le cours d'une pensée se découvrant (voir Favriaud, Vinsonneau \& Poletto, 2017).

En somme, rien de tout cela ne semble possible sans la construction d'un milieu d'apprentissage propice ni la création par le maitre des situations didactiques et des gestes professionnels favorables.

\section{L'écopoésie : un facteur d'apprentissage décisif ?}

57 Le concept d'écopoésie a été inventé par le groupe Alep dans les années 2010 comme milieu d'apprentissage de la lecture et de la langue avec les arts et la poésie, empruntant à l'ergonomie tant du travail (G. Sensevy) que de l'enseignement-apprentissage (A. Jorro, sur les pas de M. de Certeau et de C. Lévi-Strauss) en intégrant l'éthique de la création (H. Meschonnic) et de la relation (S. Martin), et en faisant le lien entre l'imaginaire le plus singulier des apprenants et leur réflexivité sur la langue et les formes sémiotiques (Favriaud, Vinsonneau \& Poletto, 2017, p. 223-232).

Les gestes professionnels du maitre y tiennent une place centrale, avec notamment les gestes de sécurisation et de lâcher-prise, d'« altérité » (Liu, 2018), de saisie de l'occasion bonne (kaïros d'A. Jorro) ou mauvaise (Favriaud et al., 2009, p. 175-199) et le geste clé de voûte de bonification, consistant à amener les élèves à un niveau réflexif supérieur (ibid., p. 184).

59 Le double geste de sécurisation et d'aide au lâcher-prise est essentiel dans toute démarche de création, qui risque d'abord de déstabiliser les apprenants; on le retrouve notamment dans la « diction expérimentée et musiquée » (Favriaud et al., 2010, p. 49-61) et dans les ateliers d'écriture ; dans notre échantillon, le lâcher-prise est exemplifié par $\mathrm{E}$ réassemblant ${ }^{7}$, avec la bienveillance du maitre, les premiers mots des vers pour en construire un nouveau ou lisant étonnamment un vers à l'envers. Le geste d'altérité concourt alors à la bienveillance des pairs et à la discussion constructive de ceux-ci réunis en comité éditorial, à l'occasion des créations offertes. Le geste de kaïros porte l'énergie de rebond du maitre saisissant l'occasion qui le surprend lui-même (Voir le «Ah ! oui !» de surenchérissement de $\mathrm{D}$ conversant avec $\mathrm{P}$ ), valorise l'intervention de l'élève et pousse dans de nouvelles voies, d'expérimentation ou de réflexion.

Le geste majeur de bonification part lui aussi des propositions de l'élève pour amener celui-ci à un niveau de réflexion et de conceptualisation supérieure, dans ce que L. Vygotski appelait la « zone proximale de développement », dont l'étendue pourrait être alors plus ambitieusement large qu'on ne le prétend généralement, quand l'imaginaire est convoqué. $\mathrm{D}$ met en œuvre ce geste avec $\mathrm{P}$ quand elle fait comparer poésie et récit ordinaire ; la comparaison amène la réflexivité, avec les possibilités soit de distinction, soit de généralisation à un niveau de classification supérieure. L'entretien d'élucidation, duel ou collectif, pourrait devenir le genre d'activité majeur de cette aide à la réflexivité et à l'abstraction. 


\section{L'écopoésie d'Alep augmentée : le modèle 2018 de D. Liu}

61 À la suite des travaux doctoraux de D. Liu, le facteur humain pourrait être encore renforcé, non seulement dans le rapport maitre-élève, très présent dans le modèle antérieur, mais dans le rapport élève-élève, élève-parents, élèves-correspondants extérieurs (autres classes, poètes).

Ainsi, B non seulement suit les consignes magistrales d'activités (lire à haute voix, recopier un extrait de poème dans le carnet de poésie, écrire son propre poème et préparer son anthologie personnelle), mais il participe à des créations collectives, qui trouvent probablement leur origine dans les correspondances avec des classes extérieures et avec des poètes publiés. Le plus original est l'extension de l'écopoésie à la sphère familiale, partenaire indispensable de la littératie : $\mathrm{B}$ lit des poèmes à ses parents, et à lui-même avant de trouver le sommeil. La poésie est devenue une écologie de vie, de rapport aux autres et à soi-même. On aboutit alors au schéma d'activités suivant.

Figure 1. Activités et relations en écopoésie (D. Liu)

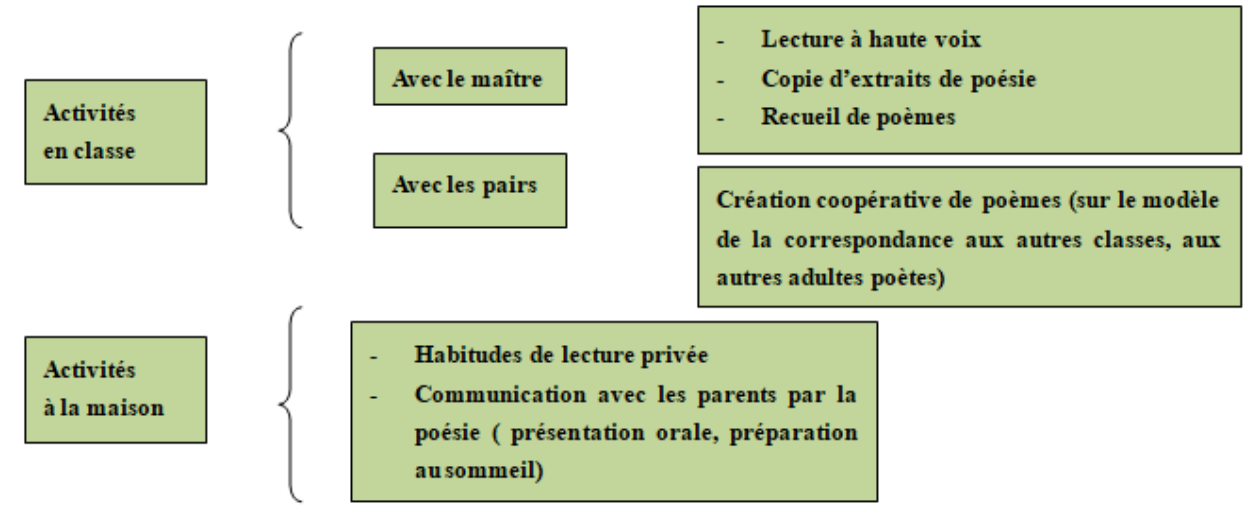

63 Nous proposons ainsi un aménagement du modèle écopoétique précédent, figurant l'entrée des élèves dans la littératie aux cycles 1 et 2 (qui pourrait avoir son pendant aux cycles 3 et 4 , autour de la grammaire et des genres textuels). 


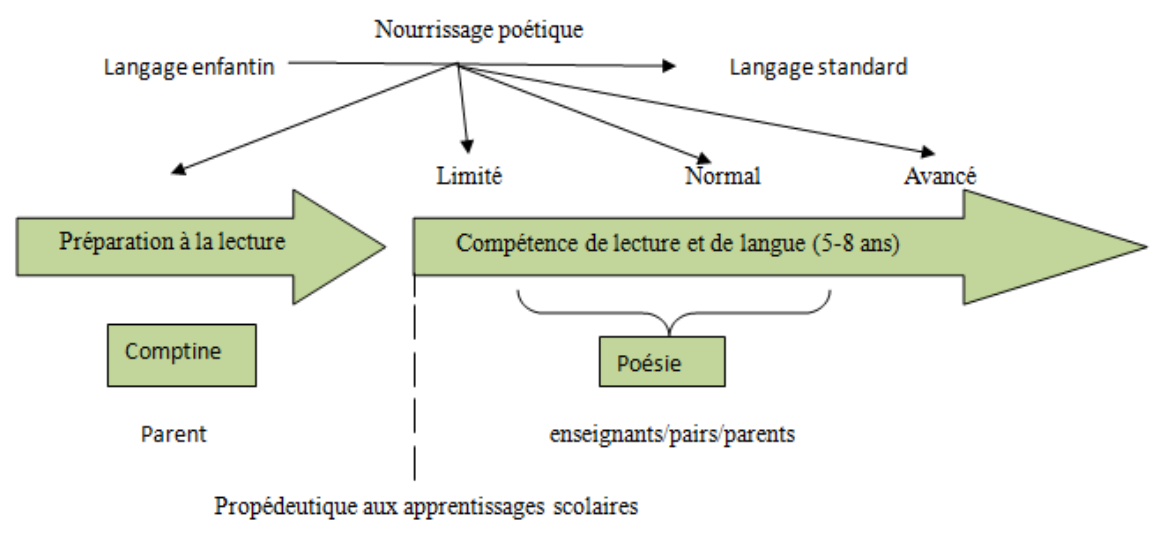

Donc, le modèle simple d'apprentissage de la lecture au cycle 2, promu par le ministre de l'Éducation nationale J.-M. Blanquer, semble vouer, une fois de plus, une partie importante des élèves de 5-8 ans à l'échec. Certes la place attribuée au principe phonologique est juste, mais trop exclusive des morphologie, syntaxe et sémantique qui demanderaient au contraire une démarche épi et méta conjointe. De plus, la compétence de lecture, ne faisant non plus le minutieux rapport avec les schèmes cognitifs généraux et avec l'imaginaire - singulier de chaque enfant et culturel de ses diverses appartenances - ne pourra s'installer aisément que pour les enfants déjà structurés par un milieu familial dynamique et équilibré, où la langue est plus qu'un médiateur ordinaire, mais déjà une ressource de vie, imaginaire et réflexive.

Or, les comptines et les poèmes - par leur double mouvement de régression-réparation et d'anticipation dynamique au plan psychologique - par leur effet d'écoute, de loupe et de questionnement au plan linguistique - permettraient le nouage des deux plans, mais à des conditions ignorées dans les années 1970-2000, que la recherche didactique et anthropologique devrait explorer systématiquement: des genres d'activité renouvelés, plus implicatifs, créatifs et délibératifs, engendrant l'émotion, le partage, la discussion et la négociation des valeurs, plus linguistiques et discursives que morales; un milieu éthique, artistique, pré-philosophique, l'écopoésie, fondé sur le "commerce» des différences au sein de la communauté éducative ; une attitude professionnelle du maitre qui, ne pouvant relever de la spécialité littéraire, ne pourrait se situer qu'au niveau éthique, écologique, voire artistique.

Si nous essayons de construire le lien indissoluble et fondateur entre imaginaire et réflexivité au service de la lecture, de la langue et de la vie, c'est que nous estimons, selon le témoignage même des enfants, que l'éducation ne peut se faire sans art ni abstraction. Le métier d'enseignant serait pauvre et harassant s'il n'était qu'une technique; il doit être requalifié en art, soucieux de soi-même, des autres et du monde. "L'agir professionnel » d'A. Jorro (2006) peut devenir un " agir poétisé », surtout si l'on entend la parole échangée avec D. Maximin : « les enfants en difficulté SONT des poètes »" 


\section{BIBLIOGRAPHIE}

ANZIEU, D. (1994). Le penser, du moi peau au moi pensant. Paris : Dunod.

BetTelheim, B. (1976). La Psychanalyse des contes de fée. Paris : Robert Laffont.

BONNEFoY, Y. (2016). L'écharpe rouge. Paris : Mercure de France.

BOUCHet (du), A. (1979). L'incohérence. Paris : Fata Moragana.

BRYANT, P. E. et al. (1989). « Nursery rhymes, phonological skills and reading ». Journal of Child

Language 16 (02), p. 407-428.

Chamoiseau, P. (2016). La matière de l'absence. Paris : Éd. Le Seuil.

CHENG, F. (2016). De l'âme. Paris : A. Michel.

CHRISTIN, A.-M. (1995). L'image écrite ou la déraison graphique. Paris : Flammarion.

CHRISTIN, A.-M. (2011). L'invention de la figure. Paris : Flammarion.

Coulon, A. (2014) [1987]. L'Ethnométhodologie. Paris : Presses universitaires de France.

CREECH, S. (2003) [2001]. J'aime pas la poésie! Trad. de l'anglais par A. Krief. Paris : Gallimard.

DEHAENE, S. (dir.) (2011). Apprendre à lire. Des sciences cognitives à la salle de classe. Paris : O. Jacob.

DIATKINE, R. (1990). « Développement psychique et transmission culturelle ». Enfance 43 (1-2),

p. 25-32. Enligne : https://www.persee.fr/doc/enfan_0013-7545_1990_num_43_1_1910.

FAVRIAUD M. (2012), «A l'affut de la parole : poétique, ponctuation et syntaxe d'André du Bouchet ». In : Collot, M. \& Léger, J.-P. (éds), Présence d'André du Bouchet. Paris : Hermann, p. 163-178.

FAVRIAUD M. et al. (2009). « Nourrissage, amorçage et gestes professionnels dans la production écrite au cycle 2 de l'école primaire ». Repères 40, p. 175-199. En ligne : https:// journals.openedition.org/reperes/340.

FAVRIAUD, M. (2016). « Poésie à l'école primaire : à la marge ou au cœur de la littératie ?». In : Petitjean, A. (éd.), Didactiques du français et de la littérature. Metz : Université de Lorraine, p. 521-536.

FAVRIAUD, M. et al. (2010) « Ce que les élèves, au cycle 2, vivent et pensent avec la poésie intéresset-il la poétique autant que la didactique ? ». Le Français aujourd'hui 169, p. 49-61. En ligne : https:// www.cairn.info/revue-le-francais-aujourd-hui-2010-2-page-49.htm.

FAVRIAUD, M. et al. (2011). « Le poème du lecteur : mémorisation, imagination, compréhension - et image de soi ». In : Langlade, G. \& Mazauric, C. (éds), Le texte du lecteur. Bruxelles : P. Lang, p. 175-192.

FAVRiaud, M., VInSONNEAU, M. \& PoletTo, M. (2017). Les chemins de poésie d'Alep. Poétique et didactique du dire-lire-écrire à l'école primaire. Limoges : Lambert-Lucas.

GAUTHIER, J.-M. \& LeJEUnE, C. (2008). « Les comptines et leur utilité dans le développement de l'enfant ». Neuropsychiatrie de l'enfance et de l'adolescence 56, p. 413-421. 
GOIGOUX, R. \& CÈBE, S. (2006). Apprendre à lire à l'école. Paris : Retz.

GOIGOUX, R. \& CÈBE, S. (2013). Lectorino et Lectorinette. Paris : Retz.

GOMBERT J.-É. (2003). « L'apprentissage des codes grapho-phonologique et grapho-sémantique en lecture ». In : ROMDHANE, M. N., GOMBERT, J.-É. \& BELJOUZA, M. (dirs), L'apprentissage de la lecture.

Rennes: Presses universitaires de Rennes, p. 19-34.

HERBAUTS, A. (2006). De temps en temps. Noville-sur-Mehaigne : Esperluète.

JORRO, A. (1998). «L'inscription des gestes professionnels dans l'action ». En Question 19. En ligne :

https://halshs.archives-ouvertes.fr/halshs-00112344/document.

JORRO, A. (2006). «L'agir professionnel de l'enseignant ». Séminaire de recherche du Centre de

Recherche sur la formation. Paris : CNAM. En ligne : https://halshs.archives-ouvertes.fr/

halshs-00195900/document.

LEBOVICI, S., DiATKINE, R. \& SOULÉ, M. (dirs) (2004) [1985]. Nouveau traité de psychiatrie de l'enfant et de l'adolescent. Paris : Presses universitaires de France.

LEGENDRE, F. (1984). « Bruno Duborgel. Imaginaire et pédagogie ». Bulletin des bibliothèques de France 3, p. 259. En ligne : http://bbf.enssib.fr/consulter/bbf-1984-03-0259-003.

LIU, D. (2018). The role and effect of poetry in reading for 5-8 years old. Thèse en didactique et littérature moderne. Université Toulouse Jean-Jaurès.

LUCA, G. (1985). Héros-limite. Paris : Corti.

MACÉ, M. (2016). Styles. Critique de nos formes de vie. Paris : Gallimard.

MARTIN, S. (2017). Voix et relation. Une poétique de l'art littéraire où tout se rattache. Taulignan :

M. Delabre.

MESCHONNIC, H. (1973). Pour la poétique III. Une parole écriture. Paris : Gallimard.

MESCHONNIC, H. (1995). Politique du rythme. Politique du sujet. Lagrasse : Verdier.

QUIGNARD, P. (2017). Performances de ténèbres. Paris : Galilée.

RICÆUR, P. (1975). La métaphore vive. Paris : Éd. Le Seuil.

SPRENGER-CHAROLLES, L. (1986). « Rôle du contexte linguistique, des informations visuelles et phonologiques dans la lecture et son apprentissage ». Pratiques 52, p. 9-27. En ligne : https:// www.persee.fr/doc/prati_0338-2389_1986_num_52_1_1406/.

VYGoTSKI, L. (1997). Pensée et Langage. Paris : Éd. La Dispute.

\section{NOTES}

1. D. Liu parle d' « écopoétrie ».

2. Les IO 1995 de l'École maternelle en parlaient plus: tcherome.fr/uploads/fichiers institutionnels/Instructions.

3. Le poète $\mathrm{A}$. du Bouchet met au jour trois modes de structuration du discours poétique : le détour, le demi-tour et le couper court (Favriaud, 2012, p. 173-178).

4. Les élèves de GSM sont ici sous-représentés pour des raisons de place.

5. M. Poletto a participé au groupe Alep (rattaché à LLA-Créatis de l'université de Toulouse JeanJaurès) et à son ouvrage collectif, Les chemins de poésie d'Alep, (Favriaud, Vinsonneau \& Poletto, 2017) ; sa classe a été le terrain majeur de la recherche doctorale de D. Liu, soutenue en 2018. 
6. Soulignement de M. Favriaud et D. Liu.

7. Le réassemblage est noté par A. Bernadet dans cette livraison même comme une opération (grammaticale ?) de poésie promue par É. Benveniste dans son Baudelaire.

8. Cette réflexion orale nous a été tenue deux fois par D. Maximin en marge du colloque, Les Arts face à l'histoire (M.-H. Popelard, dir) (2004), L'Atelier des Brisants. Ce propos trouve plusieurs illustrations dans l'œuvre romanesque de Maximin.

\section{RÉSUMÉS}

Cet article, constatant l'échec de la politique de l'apprentissage de la lecture depuis les années 1980, essaie de promouvoir le rôle de la poésie comme accompagnatrice d'une méthode systématique inspirée des résultats des sciences cognitives et de la linguistique. Il montre que les poèmes, dans le sillage des comptines, permettraient de faciliter le saut symbolique et cognitif que représente cette seconde symbolisation linguistique, en faisant le rapport entre l'imaginaire du sujet apprenant et son questionnement plus abstrait sur le fonctionnement de la langue. Mais cette facilitation de la poésie ne peut idéalement se faire qu'à des conditions strictes d'étayage et de milieu d'apprentissage, l'écopoésie.

This article, noting the failure of the policy of learning to read since the eighties, tries to promote the role of poetry as a companion to a systematic method inspired by the laws of cognitive science and linguistics. It shows that poems, in the wake of nursery rhymes, would facilitate the symbolic and cognitive jump represented by this second linguistic symbolization, making the connection between the imagination of the learner and his more abstract questioning on the functioning of the language. But this facilitation of poetry can ideally be done only under strict conditions of support and environment of learning, "écopoésie"(ecopoesis).

\section{INDEX}

Mots-clés : apprentissage de la lecture, poésie, comptine, imaginaire, réflexivité, épilinguistique, métalinguistique, écopoésie, gestes professionnels, éthique

Keywords : learning to read, poetry, nursery rhyme, imaginary, reflexivity, epilinguistics, metalinguistics, écopoésie, professional gestures, ethics

\section{AUTEURS}

\section{MICHEL FAVRIAUD}

Université Toulouse - Jean Jaurès, LLA-Créatis, EA 4152, F-31000, France

DAN LIU

Southwestern University, CN-400715, Chine 